DFUB 02/05

University of Bologna, July 2002

\title{
Final results of magnetic monopole searches with the MACRO experiment
}

\author{
The MACRO Collaboration \\ M. Ambrosio ${ }^{12}$, R. Antolini ${ }^{7}$, G. Auriemma ${ }^{14, a}$, D. Bakari ${ }^{2,17}$, A. Baldini ${ }^{13}$, G. C. Barbarino ${ }^{12}$, B. C. Barish ${ }^{4}$, \\ G. Battistoni ${ }^{6, b}$, Y. Becherini ${ }^{2}$, R. Bellotti ${ }^{1}$, C. Bemporad ${ }^{13}$, P. Bernardini ${ }^{10}$, H. Bilokon ${ }^{6}$, C. Bloise ${ }^{6}$, C. Bower ${ }^{8}$, \\ M. Brigida ${ }^{1}$, S. Bussino ${ }^{18}$, F. Cafagna ${ }^{1}$, M. Calicchio ${ }^{1}$, D. Campana ${ }^{12}$, M. Carboni ${ }^{6}$, R. Caruso ${ }^{9}$, S. Cecchini ${ }^{2, c}$, \\ F. Cei ${ }^{13}$, V. Chiarella ${ }^{6}$, T. Chiarusi ${ }^{2}$, B. C. Choudhary ${ }^{4}$, S. Coutu ${ }^{11, i}$, M. Cozzi $^{2}$, G. De Cataldo ${ }^{1}$, H. Dekhissi $^{2}, 17$, \\ C. De Marzo ${ }^{1}$, I. De Mitri10, J. Derkaoui ${ }^{2,17}$, M. De Vincenzi ${ }^{18}$, A. Di Credico ${ }^{7}$, O. Erriquez ${ }^{1}$, C. Favuzzi ${ }^{1}$, C. Forti $^{6}$, \\ P. Fusco ${ }^{1}$, G. Giacomelli ${ }^{2}$, G. Giannini ${ }^{13, d}$, N. Giglietto ${ }^{1}$, M. Giorgini ${ }^{2}$, M. Grassi ${ }^{13}$, A. Grillo ${ }^{7}$, F. Guarino ${ }^{12}$, \\ C. Gustavino ${ }^{7}$, A. Habig ${ }^{3, p}$, K. Hanson ${ }^{11}$, R. Heinz ${ }^{8}$, E. Iarocci ${ }^{6, e}$, E. Katsavounidis ${ }^{4, q}$, I. Katsavounidis ${ }^{4, r}$, \\ E. Kearns ${ }^{3}$, H. Kim ${ }^{4}$, S. Kyriazopoulou ${ }^{4}$, A. Kumar ${ }^{19,2}$, E. Lamanna ${ }^{14, l}$, C. Lane ${ }^{5}$, D. S. Levin ${ }^{11}$, P. Lipari ${ }^{14}$, \\ N. P. Longley ${ }^{4, h}$, M. J. Longo ${ }^{11}$, F. Loparco ${ }^{1}$, F. Maaroufi, ${ }^{2,17}$, G. Mancarella ${ }^{10}$, G. Mandrioli ${ }^{2}$, S. Manzoor ${ }^{2, n}$, \\ A. Margiotta ${ }^{2}$, A. Marini ${ }^{6}$, D. Martello ${ }^{10}$, A. Marzari-Chiesa ${ }^{16}$, D. Matteuzzi ${ }^{2}$, M. N. Mazziotta ${ }^{1}$, D. G. Michael ${ }^{4}$, \\ P. Monacelli ${ }^{9}$, T. Montaruli ${ }^{1}$, M. Monteno ${ }^{16}$, S. Mufson ${ }^{8}$, J. Musser ${ }^{8}$, D. Nicolò ${ }^{13}$, R. Nolty ${ }^{4}$, C. Orth ${ }^{3}$, \\ G. Osteria ${ }^{12}$, O. Palamara ${ }^{7}$, V. Patera ${ }^{6, e}$, L. Patrizii ${ }^{2}$, R. Pazzi ${ }^{13}$, C. W. Peck ${ }^{4}$, L. Perrone ${ }^{10}$, S. Petrera ${ }^{9}$, \\ P. Pistilli ${ }^{18}$, V. $\mathrm{Popa}^{2, g}$, A. Rainò ${ }^{1}$, J. Reynoldson 7 , F. Ronga ${ }^{6}$, A. Rrhioua ${ }^{2,17}$, C. Satriano ${ }^{14, a}$, E. Scapparone ${ }^{7}$, \\ K. Scholberg ${ }^{3, q}$, A. Sciubba ${ }^{6, e}$, P. Serra ${ }^{2}$, M. Sioli ${ }^{2}$, G. Sirri ${ }^{2}$ M. Sitta ${ }^{16, o}$, P. Spinelli ${ }^{1}$, M. Spinetti ${ }^{6}$, M. Spurio ${ }^{2}$,

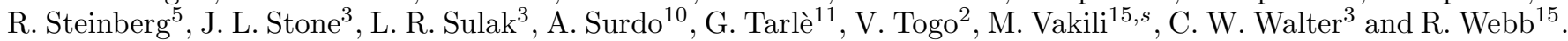

1. Dipartimento di Fisica dell'Università di Bari and INFN, 70126 Bari, Italy

2. Dipartimento di Fisica dell'Università di Bologna and INFN, 40126 Bologna, Italy

3. Physics Department, Boston University, Boston, MA 02215, USA

4. California Institute of Technology, Pasadena, CA 91125, USA

5. Department of Physics, Drexel University, Philadelphia, PA 19104, USA

6. Laboratori Nazionali di Frascati dell'INFN, 00044 Frascati (Roma), Italy

7. Laboratori Nazionali del Gran Sasso dell'INFN, 67010 Assergi (L'Aquila), Italy

8. Depts. of Physics and of Astronomy, Indiana University, Bloomington, IN 47405, USA

9. Dipartimento di Fisica dell'Università dell'Aquila and INFN, 67100 L'Aquila, Italy

10. Dipartimento di Fisica dell'Università di Lecce and INFN, 73100 Lecce, Italy

11. Department of Physics, University of Michigan, Ann Arbor, MI 48109, USA

12. Dipartimento di Fisica dell'Università di Napoli and INFN, 80125 Napoli, Italy

13. Dipartimento di Fisica dell'Università di Pisa and INFN, 56010 Pisa, Italy

14. Dipartimento di Fisica dell'Università di Roma "La Sapienza" and INFN, 00185 Roma, Italy

15. Physics Department, Texas A\&M University, College Station, TX 77843, USA

16. Dipartimento di Fisica Sperimentale dell'Università di Torino and INFN, 10125 Torino, Italy

17. L.P.T.P, Faculty of Sciences, University Mohamed I, B.P. 524 Oujda, Morocco

18. Dipartimento di Fisica dell'Università di Roma Tre and INFN Sezione Roma Tre, 00146 Roma, Italy

19. Department of Physics, Sant Longowal Institute of Engg. \& Tech., Longowal 148 106, India

a Also Università della Basilicata, 85100 Potenza, Italy

$b$ Also INFN Milano, 20133 Milano, Italy

c Also IASF/CNR sez. Bologna, 40129 Bologna, Italy

$d$ Also Università di Trieste and INFN, 34100 Trieste, Italy

e Also Dipartimento di Energetica, Università di Roma, 00185 Roma, Italy

$g$ Also Institute for Space Sciences, 76900 Bucharest, Romania

$h$ Macalester College, Dept. of Physics and Astr., St. Paul, MN 55105

$i$ Also Department of Physics, Pennsylvania State University, University Park, PA 16801, USA

lAlso Dipartimento di Fisica dell'Università della Calabria, Rende (Cosenza), Italy

$n$ Also RPD, PINSTECH, P.O. Nilore, Islamabad, Pakistan

$o$ Also Dipartimento di Scienze e Tecnologie Avanzate, Università del Piemonte Orientale, Alessandria, Italy

$p$ Also U. Minn. Duluth Physics Dept., Duluth, MN 55812

$q$ Also Dept. of Physics, MIT, Cambridge, MA 02139

$r$ Also Intervideo Inc., Torrance CA 90505 USA 
$s$ Also Resonance Photonics, Markham, Ontario, Canada

Received: July 2002

Abstract. We present the final results obtained by the MACRO experiment in the search for GUT magnetic monopoles in the penetrating cosmic radiation, for the range $4 \times 10^{-5}<\beta<1$. Several searches with all the MACRO sub-detectors (i.e. scintillation counters, limited streamer tubes and nuclear track detectors) were performed, both in stand alone and combined ways. No candidates were detected and a $90 \%$ Confidence Level (C.L.) upper limit to the local magnetic monopole flux was set at the level of $1.4 \times 10^{-16} \mathrm{~cm}^{-2} \mathrm{~s}^{-1}$ $\mathrm{sr}^{-1}$. This result is the first experimental limit obtained in direct searches which is well below the Parker bound in the whole $\beta$ range in which GUT magnetic monopoles are expected. 


\section{Introduction}

Within the framework of Grand Unified Theories (GUT) of the strong and electroweak interactions, supermassive magnetic monopoles (MMs) with masses $m \sim 10^{17} \mathrm{GeV} / \mathrm{c}^{2}$ could have been produced in the early Universe as intrinsically stable topological defects at the phase transition in which a simple gauge symmetry left an unbroken $\mathrm{U}(1)$ group [1]. At our time they can be searched for in the penetrating cosmic radiation as "fossil" remnants of that transition. The detection of such a particle would be one of the most spectacular confirmation of GUT predictions.

The velocity range in which GUT magnetic monopoles should be sought spreads over several decades. If sufficiently heavy $\left(m \gtrsim 10^{17} \mathrm{GeV} / \mathrm{c}^{2}\right)$, GUT magnetic monopoles would be gravitationally bound to the galaxy with a velocity distribution peaked at $\beta=v / c \simeq 10^{-3}$ [1]. MMs trapped around the Earth or the Sun are expected to travel with $\beta \simeq 10^{-5}$ and $\simeq 10^{-4}$, respectively.

Intermediate mass MMs $\left(10^{15}<m<10^{17}\right)$ could have been produced in later phase transitions in the early Universe at a lower energy scale [2]; lighter magnetic monopoles, with masses around $10^{7} \div 10^{15} \mathrm{GeV} / \mathrm{c}^{2}$, would be accelerated to relativistic velocities in one or more coherent domains of the galactic magnetic field, or in the intergalactic field, or in several astrophysical sites like a neutron star [3].

Theory does not provide definite predictions on the magnetic monopole abundance. However, by requiring that MMs do not short-circuit the galactic magnetic field faster than the dynamo mechanism can regenerate it, a flux upper limit can be obtained. This is the so-called Parker bound $\left(\sim 10^{-15} \mathrm{~cm}^{-2} \mathrm{~s}^{-1} \mathrm{sr}^{-1}\right.$ [4]), whose value sets the scale of the detector exposure for MMs search. The original Parker limit was re-examined to take into account the almost chaotic nature of the galactic magnetic field, with domain lengths of about $1 \div 10 \mathrm{kpc}$; the limit become mass-dependent. An Extended Parker Bound (EPB) at the level of $1.2 \times 10^{-16}\left(\mathrm{~m} / 10^{17}\right) \mathrm{cm}^{-2} \mathrm{~s}^{-1} \mathrm{sr}^{-1}$ was obtained [32].

MACRO was a large multipurpose underground detector located in the Hall B of the Laboratori Nazionali del Gran Sasso (Italy); it was optimized for the search for GUT magnetic monopoles with velocity $\beta \geq 4 \times 10^{-5}$ and with a sensitivity well below the Parker bound. The detector, which took data up to December 2000, is fully

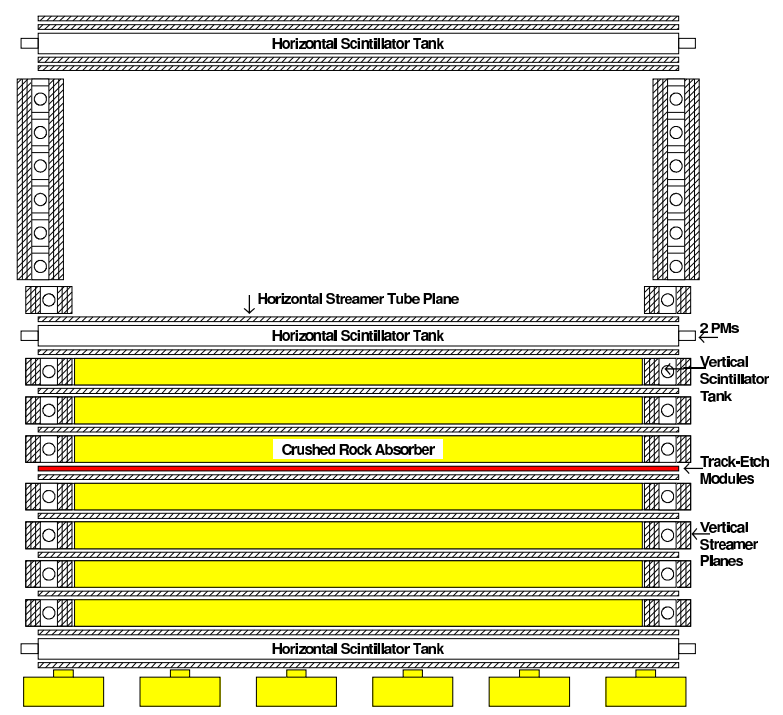

Fig. 1. A cross-sectional view of the MACRO detector showing the layout of the 3 sub-detectors and of the rock absorber.

described in 5.6 . It was arranged in a modular structure, it was divided into six $12.6 \times 12 \times 9.3 \mathrm{~m}^{3}$ sections referred to as SuperModules (SM), each one with separate mechanical structure and electronics readout. The detector's global dimensions were $76.5 \times 12 \times 9.3 \mathrm{~m}^{3}$ and its total acceptance for an isotropic flux of particles was $\sim 10,000 \mathrm{~m}^{2} \mathrm{sr}$.

Redundancy and complementarity were the primary features in designing the experiment. Since we could not reasonably expect more than a few MMs during the detector lifetime, we deemed crucial to have multiple signatures and the ability to perform cross checks among various parts of the apparatus. To accomplish this, the detector consisted of three independent sub-detectors: liquid scintillation counters, limited streamer tubes and nuclear track detectors, each of them with dedicated and independent hardware. In Fig. 11 a cross sectional view of the apparatus is shown. Also visible are the seven horizontal absorber layers (which set at $\sim 1 \mathrm{GeV}$ the minimum energy threshold for throughgoing muons); notice the separation of the detector into a lower and an upper detector (the attico).

The background that magnetic monopole searches have to fight with is mainly due to muons of the cosmic 
radiation and natural radioactivity. Thus large detectors have to be installed in underground laboratories. At Gran Sasso the minimum thickness of the rock overburden above the detector is $3150 \mathrm{hg} / \mathrm{cm}^{2}$. The cosmic radiation muon flux in Hall $\mathrm{B}$ is $\sim 1 \mathrm{~m}^{-2} \mathrm{~h}^{-1}$, almost a factor $10^{6}$ smaller than that at the surface (only muons with minimum energy of $\simeq 1.3 \mathrm{TeV}$ can cross the mountain and reach $\mathrm{MACRO}$ ) with an average muon residual energy of $\sim 300 \mathrm{GeV}$ [7].

The signatures of the passage of a GUT magnetic monopole across the detector depend strongly on its velocity [1,8]. For this reason, different hardware systems were designed and operated to give optimum sensitivity in different $\beta$ values. Different analysis strategies were also adopted, depending on the $\beta$ range of interest and the subdetector(s) used; the entire MM $\beta$ range $\left(4 \times 10^{-5}<\right.$ $\beta<1$ ) was thus covered. In the different analyses we took into account both the MM signatures and the background characteristics.

The unique property of a fast magnetic monopole with $\beta \geq 10^{-2}$ 1], 8 is its large ionization power compared either to the considerably slower magnetic monopoles or to the minimum ionizing electrically charged particles. The searches for fast MMs look for large energy releases in the detectors; the background is mainly due to high energy muons with or without an accompanying electromagnetic shower. On the other hand, slow magnetic monopoles should leave small signals spread over a large time window; a $\beta \sim 10^{-4}$ monopole could have a Time of Flight (ToF) across the detector as large as $\sim 1 \mathrm{~ms}$. This implies the use of specific analysis procedures that allow the rejection of the background mainly due to radioactivity induced hits and possible electronic noise.

In this paper we report the final results of several magnetic monopole searches performed with MACRO (Sect.2); some early results were already published in [9]. In each Section we discuss the analysis criteria together with the results of each search; technical details may be found in various papers fully describing the procedures and their application to the first data samples 10,11,12,13,14. In Sect. 3 the result of the combination of all the various searches is reported. In order to compare the MACRO results to those of other experiments or to theoretical models, we present upper limits for an isotropic flux of bare MMs with magnetic charge equal to one Dirac charge $g=g_{D}=e / 2 \alpha$, (where $e$ is the electron charge and $\alpha$ the fine structure constant) and nucleon decay catalysis cross section smaller than $1 \mathrm{mb}[1]$. These aspects are discussed in Sect. 1 the conclusions are given in Sect. 5. A dedicate analysis for the search for magnetic monopoles accompanied by one or more nucleon decays along their path was also performed. The results are reported in a separate paper [37.

\section{Experimental searches}

Fig. 2. Results of the measurements of the Slow Monopole Trigger sensitivity (black squares) and of the WFD analysis efficiency (stars) plotted vs. $\beta$. The connecting lines serve only as a guide to the eye. The expected light yield of a MM is also plotted.

\subsection{Searches with Scintillators}

The MACRO liquid scintillator system was organized in three layers of horizontal and four layers of vertical counters, as shown in Fig. 1. For atmospheric muons crossing the apparatus this system provided particle position, energy deposition and ToF resolutions of about $11 \mathrm{~cm}$, $1 \mathrm{MeV}$ and $700 \mathrm{ps}$, respectively. The response of liquid scintillators to heavily ionizing particles was studied both experimentally 15 and theoretically 16,17,18 and their sensitivity to particles with $\beta$ down to $\sim 10^{-4}$ was directly measured.

\subsubsection{Wave Form Digitizer (WFD) analysis}

Slow moving magnetic monopoles (in the range $10^{-4} \lesssim \beta \lesssim$ $4.1 \times 10^{-3}$ ) were searched using dedicated hardware, the Slow Monopole Trigger (SMT) and a $200 \mathrm{MHz}$ custommade Wave Form Digitizer (WFD) system. The main goal of the SMT was to remain sensitive over the entire range of widths and amplitudes of pulses (which could as well be just a train of single photoelectron pulses lasting over several microseconds) expected for slow moving magnetic monopoles while suppressing efficiently narrow (10 $50 \mathrm{~ns}$ ) pulses due to isolated radioactivity and cosmic ray muons. The SMT by itself offered only a hit register that recorded the scintillator counters that satisfied the trigger conditions. The photomultiplier tube (PMT) pulse shape information was recorded by a custom made $200 \mathrm{MHz}$ WFD system. Redundant time-of-flight (TOF) information for every candidate event was recorded by a stand-alone TDC. The design and implementation of the triggering and recording electronics used by this search has been described in detail elsewhere [5], 6].

The heart of this slow MM search was the analysis of the digitized PMT signals as recorded by the $200 \mathrm{MHz}$ 
WFDs. Several wave form analysis methods have been employed in the search for slow MM searches in the initial data taking and results from these have been reported elsewhere [10]. In this final search the SMT hardware [5, 6] was used as the pattern recognition method for identifying a magnetic monopole candidate event [19].

The sensitivity of the trigger hardware as well as of the entire slow MM analysis was measured experimentally in situ and multiple times throughout MACRO's live time via LED light injection in each of the scintillation counters. The LED calibration system $[5.6$ allowed the generation of a magnetic monopole-like signal in the detector of arbitrary direction, ionization yield and velocity. A grid of LED pulse widths and heights allowed the generation of MM-like PMT pulses on a channel-by-channel basis. The corresponding $\beta$-range is $10^{-4}<\beta<5 \times 10^{-3}$ and the light yield from few photoelectrons up to several times the yield of a muon. The generated wave forms were recorded by the WFD system independently of whether the SMT fired or not. Then, an off-line wave form analysis (the same used to analyze the real events) established the ratio of the events that the SMT had selected over the ones expected. This yielded the trigger sensitivity as a function of the particle's velocity and it is shown in Fig. 2. More than $95 \%$ of the detector's channels exhibited efficiency above $99 \%$ for light yields greater or equal the ones defined by the curve. The generated MM-like signals were then fed into the analysis wave form selection algorithm in discussion. The stars in Fig. 2 show the analysis efficiency, when more that $99 \%$ of the simulated MM-like wave forms -corresponding to lights yields and velocities across the expected range- were successfully identified by the analysis algorithm.

There were three main instrumental sources which caused the hardware trigger to be less efficient than a software one: (a) electronics were operating at a very low threshold $(2 \mathrm{mV})$ making them susceptible to ground loops and low amplitude interference; (b) electronics suffered from intrinsic pulse streching that may overestimated pulse duration; (c) helium and hydrogen contamination in the PMT envelope increased its activity, leading sometime to overestimate of pulse duration. A software pattern recognition method corrects for them, and the efficiency of this algorithm is folded in the trigger efficiency already discussed.

Magnetic monopole candidate events were selected as follows. The hardware trigger (SMT) was required to be present in at least two of the detector faces and within $1 \mathrm{msec}$. This rejects mainly noise and radioactivity induced events. The two layers requirement reduces by $17 \%$ the detector's acceptance. The TOF requirement of $1 \mathrm{msec}$ was dictated by the hardware, namely the mode of operation of the WFD system [5]6]. The presence of the scintillator's fast particle trigger was then used as a veto. The fast coincidence $(1 \mu \mathrm{s})$ between faces required by this trigger selects particle tracks corresponding to $\beta$ well above the SMT's sensitivity (Fig. 2). It rejects mainly cosmic ray muons that triggered the SMT either due to pulse stretching or afterpulsing. A final loose cut on the hits per face (less than 4) and number of layers (less than 5) was applied to reject events induced by electronic noise, multi-muon or muon shower events. None of these requirements is expected to affect the sensitivity to slow MMs while any effect on the acceptance has been taken into account in the Monte Carlo. After applying the above cuts on the dataset of $\sim 4.75$ years of data-taking and $\sim 28$ million SMT's, there were 35901 events left. These were attributed to cosmic ray muons $(\approx 1 / 3)$ and electronic noise $(\approx 2 / 3)$ leaking out of our fast particle and loose noise/high multiplicity veto. These events were further analyzed by our wave form analysis method.

The wave form analysis required that PMT signals satisfied the pattern recognition criteria on an end-toend, face-to-face basis. 40 events survived. (There was an initial running of the detector that suffered from wave form memory saturation and effectively wave form loss. 14 events involving primarily large ionizations were allowed to pass this stage of the analysis).

The 40 remaining candidates were individually analyzed using all available information from the scintillator sub-system (the wave form shape, timing information from the wave forms, timing information from the precise muon system -ERP- and from the SMT TOF system) [6]. None of the events was compatible with the passage of a slow MM. Instead, based on information available from both the scintillator and the streamer tube sub-systems, the 40 events were classified as follows:

- 25 as bipolar electronic noise; using primarily the WFD information, they are distinguished from a genuine MM signal due to the preciable amount of positive content in the waveforms (PMTs operated with negative voltage).

- 10 as muon-induced showers; using primarily the fast muon and occasionally WFD TOF information, these events reflect fast crossing of the apparatus that failed to fire the fast particle veto.

- 4 as probably spurious electrostatic discharges of the LED system; using primarily geometrical arguments reflecting the inconsistency of the event with the passage of a monopole crossing the detector at a straight line at a constant velocity, and finally,

- the "spokesmen even". This event belongs to a particular run in which a LED-induced event (simulating a close to a realistic $\beta \sim 10^{-3} \mathrm{MM}$ ) was intentionally and secretly generated to test the efficiency of our analyses to detect a MM signal. The two analyses that should have been sensitive to the faked "spokesmen" MM (the present analysis, and the PHRASE one), found the event. The "spokesmen event" was a LED-generated event and was subject to the limitations the LED system had in generating a MM-like pulse, namely its slow rise-time.

No event was compatible with the passage of a single slow magnetic monopole. The analyzed data were collected in the period July 1995 - May 2000, corresponding to $\sim 4.3$ live-years. The detector performance during the data-taking period was monitored on a run-by-run basis. 


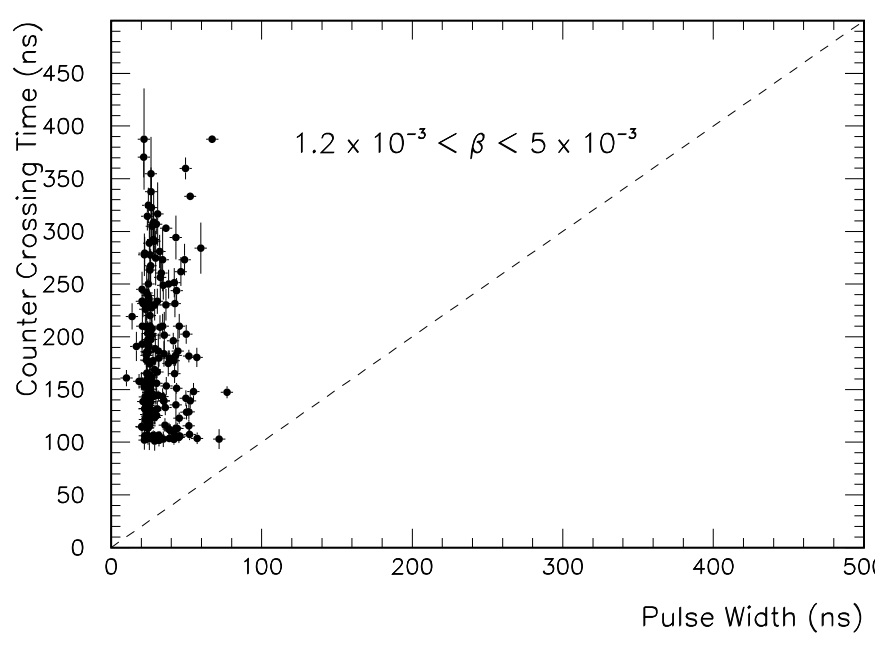

Fig. 3. Calculated crossing time in a scintillation counter versus pulse width for PHRASE monopole candidates in the range $1.2 \times 10^{-3}<\beta<5 \times 10^{-3}$ (for clarity reasons only $40 \%$ of the candidates is shown). The diagonal line indicates the minimum pulse width expected (corresponding to a MM with $\beta \simeq 5 \times 10^{-3}$ ) for $15 \mathrm{~cm}$ path length. No candidates meet or exceed this expectation.

The total exposure was $\int A d t=0.9 \times 10^{16} \mathrm{~cm}^{2} \mathrm{~s} s \mathrm{~s}$. By taking into account the trigger and analysis efficiencies we obtained a $90 \%$ C.L. flux upper limit of $2.5 \times$ $10^{-16} \mathrm{~cm}^{-2} \mathrm{~s}^{-1} \mathrm{sr}^{-1}$ for $10^{-4}<\beta<4.1 \times 10^{-3}$ (curve "WFD" in Fig. 8] [19].

\subsubsection{The PHRASE analysis}

Magnetic monopoles in the velocity range $1.2 \times 10^{-3}<$ $\beta<10^{-1}$ were searched for using the PHRASE (Pulse Height Recorder And Synchronous Encoder) system. It was a trigger and energy reconstruction processor which generated a trigger condition when the energy deposition in a scintillator was larger than a preset threshold, $E_{t h} \sim$ $7 \mathrm{MeV}[5.20 .21]$.

The energy deposition was reconstructed by integrating the photomultiplier pulses (recorded by a $100 \mathrm{MHz}$ Wave Form digitizer system), taking into account their relative amplitudes, the response functions of the scintillation counters and the liquid scintillator, photomultiplier and electronic saturation effects. All these points were studied in detail by using laser light of various intensities and atmospheric muon pulses; appropriate algorithms were developed for correcting any nonlinearity which may be present in large energy loss rates as those expected from magnetic monopoles in the analyzed $\beta$-range. The analysis technique is described in detail in [11].

The magnetic monopole candidate selection required hits in two scintillator layers and a minimum energy deposit of $10 \mathrm{MeV}$ in each of them, spread out over no more than four adjacent boxes (this cut removed most of the natural radioactivity background but not atmospheric muons). The $100 \mathrm{MHz}$ sampling frequency of the PHRASE Wave Form digitizers and the $10 \mathrm{MeV}$ energy cut defined the minimum MM velocity which this search was sensitive to: $\beta_{\min }=1.2 \times 10^{-3}$. The velocity of a particle was reconstructed by using the TOF between the crossed scintillator layers; a minimum distance of $2 \mathrm{~m}$ between the hits was required to ensure accurate timing reconstructions. The timing and position uncertainties produced a tail in the muon velocity distribution which is peaked at $\beta=1$. Atmospheric muons were rejected by setting the $\beta$ upper limit for the magnetic monopole analysis to 0.1 . Finally, a minimum path length of $15 \mathrm{~cm}$ was required in each scintillation counter (the typical path length for a crossing cosmic ray muon was $>20 \mathrm{~cm}$ ). All geometrical cuts were taking into account in computing the detector acceptance.

The candidates surviving the selection (a few thousands in $\sim 11$ years of running) were grouped in two $\beta$ overlapping sub-ranges, medium $\left(1.2 \times 10^{-3}<\beta<\right.$ $\left.5 \times 10^{-3}\right)$ and high $\left(4 \times 10^{-3}<\beta<10^{-1}\right)$ velocities. This was motivated by the fact that the MM light yield and crossing time (with respect to muons) differ significantly in these two regimes. Crossing muons released $\sim 40 \mathrm{MeV}$ in each traversed scintillation layer, with a pulse width of $\approx 35 \mathrm{~ns}$ due to the convolution between the time profile of the scintillation light emission and propagation and the PMT time jitter. The pulse width expected from a magnetic monopole in the medium $\beta$ range, corresponding to the time needed to cross a counter, is $100 \div 400 \mathrm{~ns}$. In the high $\beta$ range the expected counter crossing time is $6 \div 120 \mathrm{~ns}$; thus the corresponding pulse width is comparable to that of a muon. The expected energy loss rate, for MMs in the medium $\beta$ range, is $10 \div 30$ times larger than that of a muon, and $30 \div 60$ times for MMs in the higher $\beta$ range.

For candidates with $1.2 \times 10^{-3}<\beta<5 \times 10^{-3}$ we compared the measured pulse width with the expected counter crossing time. The counter crossing time was calculated from the measured $\beta$, assuming a minimum pathlength in the scintillation counter of $15 \mathrm{~cm}$ (Fig. (3)).

For a particle crossing the detector with the measured velocity these two numbers must be consistent. No final candidates satisfied this condition with the exception of the LED simulated "spokesmen" magnetic monopole introduced in the data (see sec. 2.1.1).

For candidates with $4 \times 10^{-3}<\beta<10^{-1}$ we compared the energy loss rate (computed with the assumption of a fixed $15 \mathrm{~cm}$ path length in the scintillator) with that expected for a MM 15,18] (see Fig. 4). The $\beta$ range was extended down to $4 \times 10^{-3}$ to obtain an independent cross-check of the rejection based on the counter crossing time criterion for the candidates at the boundary between the two sub-intervals. In no case the energy deposition of a candidate was consistent with that expected for a monopole of the same apparent velocity.

In this search magnetic monopole candidates were categorized on the basis of the streamer tube and scintillator information. No event exhibits the pattern of a single particle crossing the detector at the measured velocity; the velocity values were artifacts due to uncommon classes of events. A fraction of candidates $(\sim 10 \%)$ comes from 


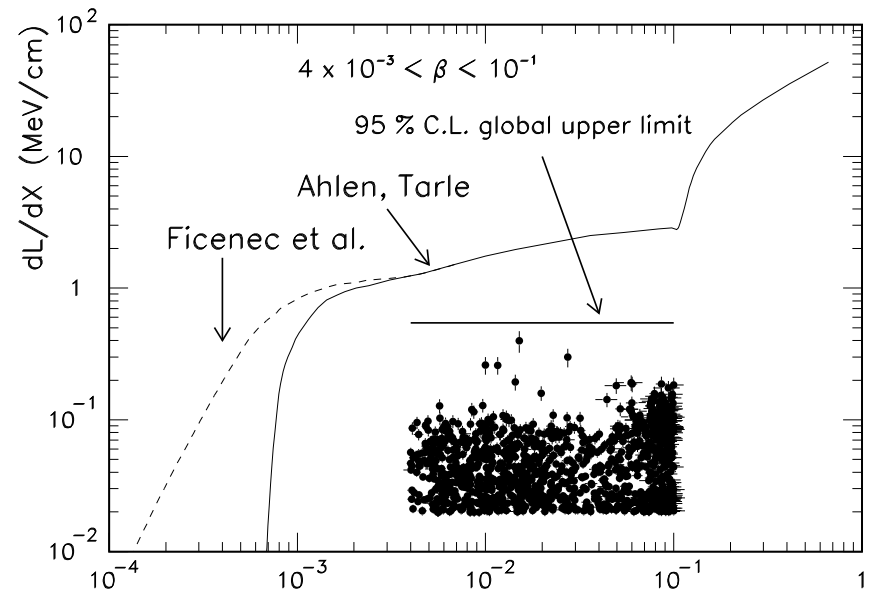

Fig. 4. Energy loss in the scintillator versus velocity for PHRASE events in the range $4 \times 10^{-3}<\beta<10^{-1}$ (for clarity only $40 \%$ of the candidates is shown). The calculated MM energy loss rates from 15, 18 are also given, as a $95 \%$ C.L. upper limit on the maximum energy loss of the candidates.

occasional timing errors, which produced an apparent low value for the velocity of a cosmic ray muon crossing the apparatus. In a larger fraction of cases $(\sim 20 \%)$, the low velocity value comes from accidental coincidences, within the allowed time window, between radioactivity hits and/or a cosmic ray muon occurring in different scintillator layers. The remaining candidates are due to cosmic ray muons which crossed a scintillator layer and stopped just before reaching a second one. The decay electron produces a hit in this second layer, with a typical $\sim 2 \mu$ s time delay (corresponding to the muon life time) with respect to the first hit.

This analysis was applied to the whole data set collected from October 1989 to June 1995 with the lower part of the detector, and with the full detector up to December 2000. The detector acceptance was computed by a Monte Carlo simulation, which takes into account the fraction of apparatus effectively in acquisition in each individual run. The total exposure was $\int A d t=1.2 \times$ $10^{16} \mathrm{~cm}^{2} \mathrm{ssr}$. By taking into account the trigger and analysis efficiencies we obtain a $90 \%$ C.L. flux upper limit $\Phi \leq 2.2 \times 10^{-16} \mathrm{~cm}^{-2} \mathrm{~s}^{-1} \mathrm{sr}^{-1}$ for $1.2 \times 10^{-3}<\beta<10^{-1}$, which is represented by curve "PHRASE" in Fig. 8.

\subsection{Searches with Streamer Tubes}

The streamer tube system was designed to be effective in the search for magnetic monopoles in a wide velocity range, $10^{-4}<\beta<1$. For this purpose we used a gas mixture containing helium and n-pentane. Helium is necessary to exploit the Drell effect [22]: the passage of a magnetic monopole with $1.1 \times 10^{-4}<\beta<10^{-3}$ through a helium atom leaves it in a excited metastable state. The n-pentane can then be ionized by collision with excited helium atoms (Penning effect). The high cross section for the processes ensures 100\% efficiency in detecting bare MMs with this gas mixture.

In the higher velocity region $\left(\beta>10^{-3}\right)$, where the assumptions used in the Drell-Penning effects do not apply, the standard ionization mechanism expected for a MM ensures an energy release several orders of magnitude higher than that due to minimum ionizing particles. The charge collected on a wire has a logarithmic dependence on the energy released inside the active cell [23,24, so that a charge measurement allowed one to distinguish between MMs and muons.

Horizontal streamer tubes were equipped with readout strips in order to provide a three dimensional event reconstruction. The memory depth of the readout electronics was large enough to store signals for monopoles with $\beta$ down to $10^{-4}$. In order to retrieve spatial coordinates, signals were shaped at $\sim 550 \mu$ s and sent to parallel-in/serialout shift register chains for readout. Analog ORs of wire signals (covering $1 \mathrm{~m}$ for central planes and $0.5 \mathrm{~m}$ for lateral planes) were fed to the Charge and Time Processor (QTP) system for arrival time and charge measurement of the streamer signals 5,5 . The trigger was based on the assumption that a heavy MM crossed the apparatus without any appreciable change in its direction and speed, due to its large kinetic energy. In designing the trigger logic, particular attention was given to avoiding the possibility that relativistic decay products from magnetic monopole induced nucleon decay, could cause dead-time in the apparatus before the MM generated the trigger [25].

Horizontal and vertical streamer tube planes were handled by two independent trigger systems. Monte Carlo simulations have shown that they were basically complementary. Two analysis were performed using data collected with the two different triggers; all streamer tubes were used for the event reconstruction. Both analyses were based on the search for single tracks and on the measurement of the particle velocity by using the time information provided by the streamer tubes (maximum time jitter $\sim 600 \mathrm{~ns}$ ) [12]. Detailed investigations were performed in order to check that both trigger logics and analysis efficiencies were independent of the particle velocity.

\subsubsection{Search with horizontal streamer tubes}

This analysis technique is described in details in [12]. The assumpition made in the trigger design allows the trigger logic to be sensitive to any massive particle able to produce a signal in the streamer tubes. The streamer tubes time resolution allows identification of slow particles by measuring their time of flight across the apparatus ("time track"). However, because of time jitter and afterpulsing in the streamer tubes, particles faster than $5 \times 10^{-3} c$ could be confused with cosmic ray muons.

The analysis was based on the search for single space tracks in both wire and strip views and on the measurement of the velocity of the candidates. The width of the temporal window is so large that on average three spurious hits (due to the $40 \mathrm{~Hz} / \mathrm{m}^{2}$ background on streamer tubes) are present in a $12 \times 12 \mathrm{~m}^{2}$ plane per event. The 
trigger logic selected events with an alignment in $z$ (the vertical coordinate) versus time $t$ of at least seven streamer tube planes in one or more of 320 time windows (called $\beta$-slices). Each $\beta$-slice covered a TOF window of 3 $\mu \mathrm{s}$ and provided a rough estimate of the particle time of flight across the apparatus.

The TOF value provided by the trigger allowed a discrimination of the streamer tube hits from a real track, from the spurious hits recorded in the $\sim 500 \mu$ s memory depth of the readout electronics. If a space track was found, a more refined alignment in the $z-t$ temporal view was required ("time track"). This rejected effectively the background from radioactivity and the background from relativistic muons combined accidentally with some radioactivity hits.

The muon provided the spatial track, while the radioactivity hits may confuse the time tracking algorithm. Such events were rejected using a cut on the measured velocity. In fact, they were tagged with both the first $\beta$ slice (that of relativistic muons) and with a higher $\beta$-slice value (that of slow particles). For particles crossing the lower detector (path length $\sim 5 \mathrm{~m}$ ) the above condition corresponds to a cut for all particles with $\beta>\beta_{\text {cut }}=$ $(5 \mathrm{~m} / 3 \mu \mathrm{s}) / \mathrm{c}=5 \times 10^{-3}$.

The sensitivity of the trigger to relativistic muons provided a simple and efficient way to estimate the overall efficiency of this analysis. Muons crossing at least seven horizontal streamer tube planes survived all the analysis steps and were only rejected by the final $\beta$-cut. The efficiency $\varepsilon_{a}$ of the analysis was then estimated by the ratio of the number of reconstructed muons to the expected number of single muons crossing at least seven planes. A further possible source of inefficiency were failures in the trigger circuits $\varepsilon_{t}$. This effect was not accounted for by the above procedure, because muons were concentrated in the first $\beta$-slice. The efficiencies $\varepsilon_{a}$ and $\varepsilon_{t}$, were completely described in [12].

This analysis used the data sample collected with the horizontal streamer tube trigger from January 1992 to September 2000, integrating a livetime of 8.1 years. The overall average efficiency was $74 \%$. The detector acceptance, computed by a Monte Carlo simulation which included geometrical and trigger requirements, was $4250 \mathrm{~m}^{2} \mathrm{sr}$.

No monopole candidates were found. For $1.1 \times 10^{-4}<$ $\beta<5 \times 10^{-3}$ the flux upper limit is $2.8 \times 10^{-16} \mathrm{~cm}^{-2} \mathrm{~s}^{-1} \mathrm{sr}^{-1}$ at $90 \%$ C.L. (Fig. 8, curve "stream. H").

\subsubsection{Search with vertical streamer tubes}

Like in the search with horizontal streamer tubes, also in this case, the reconstruction procedure started from time and space information provided by the trigger. These were used to select hits compatible in both time and position with the $\beta$-slice that fired. Using these time-position hits, we performed a complete space and time tracking of each events to reject accidental alignments; this leaves only the muon background. This was also used to evaluate the efficiencies. The spatial and temporal reconstruction algorithms are the same as those used in the horizontal

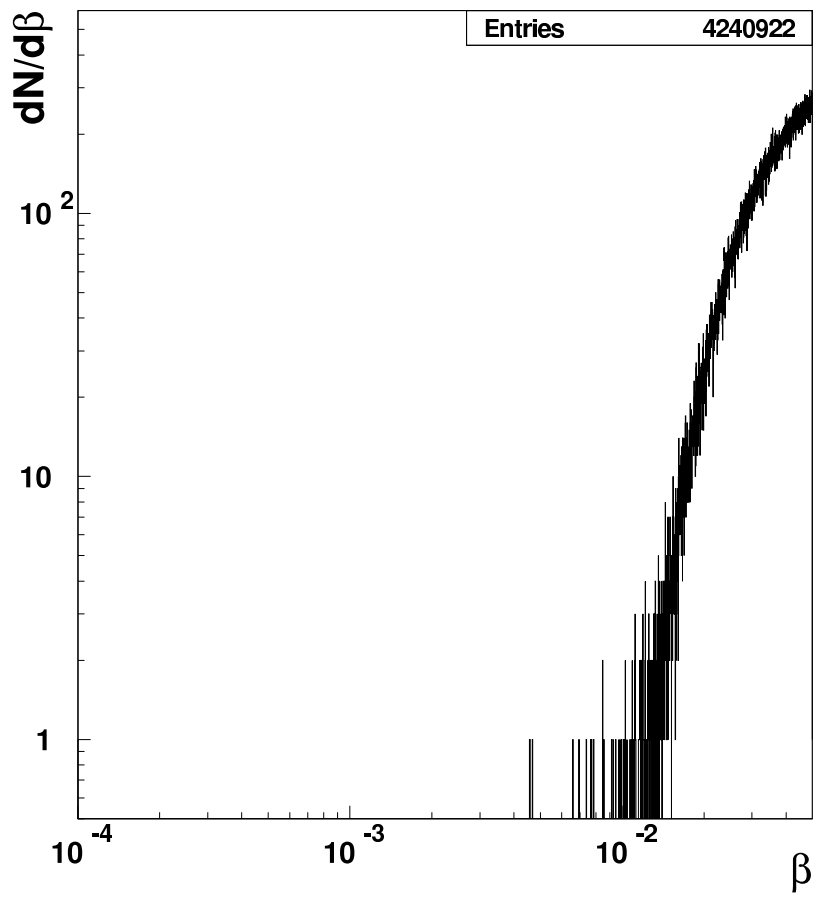

Fig. 5. The $\beta$ distribution for the candidate events reconstructed with the vertical streamer tube analysis. Events with $\beta>\beta_{c u t}^{v}=3 \times 10^{-3}$ are muons; all analyzed events are included.

analysis. On the basis of the overall event reconstruction in the 3 spatial views $(x-z, d-z, y-z)$ and in the $(t-z)$ view, it was possible to achieve the complete geometrical and temporal reconstruction of the particle track and to compute its $\beta$. The $x-z$ spatial view corresponds to the horizontal streamer tube wire view; the $d-z$ view corresponds to the horizontal streamer tube strips and the $y-z$ view corresponds to that of the lateral (vertical) streamer tube wire view. Finally the $t-z$ view is the temporal view along the vertical direction. As for the search with horizontal streamer tubes, muons are rejected if $\beta>\beta_{c u t}^{v}=3 \times 10^{-3}$ see Fig. 国.

The analysis efficiency was estimated with the same approach used for the horizontal streamer monopole search.

The search covered data from October 1994 to September 2000 for a total of 4.4 liveyears. The overall average efficiency was $70 \%$. The acceptance, estimated by Monte Carlo simulation, was $3018 \mathrm{~m}^{2} \mathrm{sr}$. The measured $\beta$ distribution, shown in Fig. 5, is broader than the one obtained from the horizontal analysis (see [12] for comparison). This limited the sensitivity of this search to the velocity range $1.1 \times 10^{-4}<\beta<3 \times 10^{-3}$. No magnetic monopole candidate was found. We thus establish an upper limit to the monopole flux $\Phi<8 \times 10^{-16} \mathrm{~cm}^{-2} \mathrm{~s}^{-1} \mathrm{sr}^{-1}$ for $10^{-4}<\beta<3 \times 10^{-3}$ (Fig. 8, curve "stream.V").

\subsection{Searches with the Track-Etch detector}

The MACRO nuclear track subdetector [5,6] covered a total area of $1263 \mathrm{~m}^{2}$ and was organized in stacks 


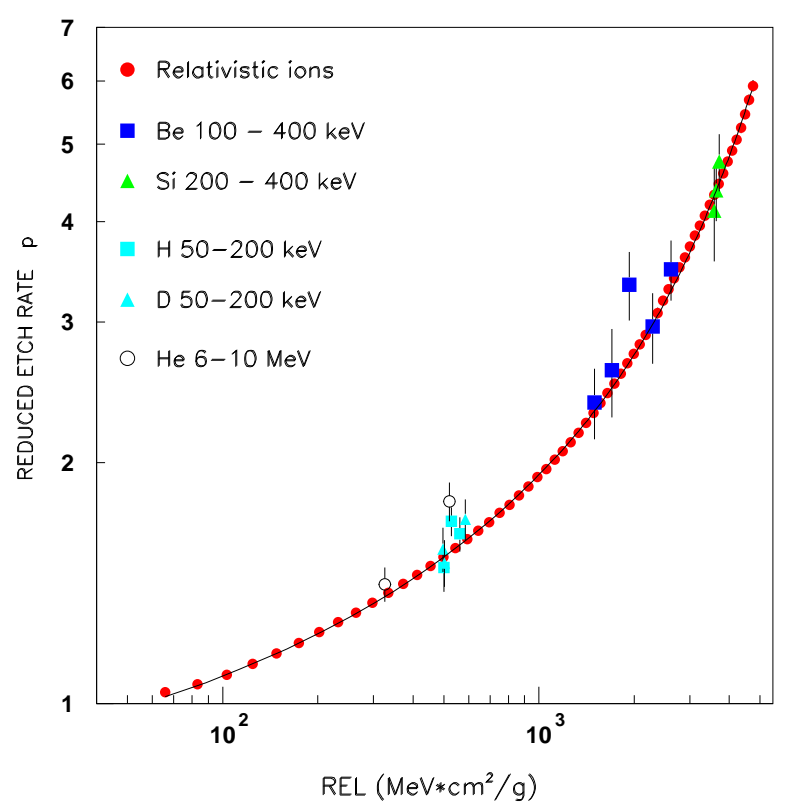

Fig. 6. Measured reduced etch rate $p$ vs REL for the CR39 track etch detector exposed to relativistic and slow ion beams; the points are the experimental data, the solid line is the best fit to the data points $(8<Z<68)$ obtained with relativistic ions.

("wagons") of $24.5 \times 24.5 \mathrm{~cm}^{2}$ consisting of three layers of CR39, three layers of Lexan and an aluminum absorber placed in an aluminized Mylar bag filled with dry air. The formation of an etchable track in a nuclear track detector is related to the Restricted Energy Loss (REL) [13]. There are two contributions to REL: the electronic energy loss $\left(S_{e}\right)$, which represents the energy transferred to the electrons, and the nuclear energy loss $\left(S_{n}\right)$, which represents the energy transferred to the nuclei in the material. In 27] it was shown that in our CR39 $S_{n}$ is as effective as $S_{e}$ in producing etchable tracks. The response of a nuclear track detector is measured by the reduced etch rate $p=v_{T} / v_{B}$, where $v_{T}$ and $v_{B}$ are the etching rates along the particle track and of the bulk material, respectively [29]. The reduced etch rate $p$ vs REL for our CR39 is shown in Fig. 6, it was measured using both relativistic and slow (down to $\beta \sim 4 \times 10^{-3}$ ) ions [27.

The CR39 allowed a search for magnetic monopoles of different magnetic charges and $\beta$. For a single Dirac charge, our CR39 is sensitive for MM in the ranges $2 \times 10^{-5}<\beta<2 \times 10^{-4}$ and $\beta>1.2 \times 10^{-3}$ [8]. Lexan has a much higher threshold making it sensitive to relativistic MMs only $\left(\beta>10^{-1}\right)$.

Our CR39 was manufactured by the Intercast Europe Co. of Parma (Italy). A specific production line was set up in order to achieve a low detection threshold, high sensitivity in a large range of energy losses, high quality of the post-etched surface after prolonged etching and stability of the detector response over long periods of time [28].
We analyzed $845.5 \mathrm{~m}^{2}$ of the MACRO CR39 track-etch sub-detector, with an average exposure time of 9.5 years. Since no candidates were found, the Lexan foils were not analyzed. The top CR39 foils were strongly etched in a $8 \mathrm{~N} \mathrm{NaOH}$ water solution at $85^{\circ} \mathrm{C}$ till their final thickness reached 300-400 $\mu \mathrm{m}$. The signal looked for was a hole or a biconical track with the two base cone areas equal within experimental uncertainties.

After etching, the foils were scanned twice, using back lighting by different operators at low magnification looking for any possible optical inhomogeneity; the double scan guarantees an efficiency close to $100 \%$ for finding an etched hole. Detected inhomogeneities were further observed with a stereo microscope and were classified either as surface defects or as particle tracks. The latter were further observed under an optical microscope with high magnification. The axes of the base-cone ellipses in the top and bottom surfaces of the foils were measured and the corresponding $p$ and incidence angle $\Theta$ computed. A track was defined as a candidate if $p$ and $\Theta$ on the top and bottom sides were equal to within $15 \%$. At a residual thickness of 300-400 $\mu \mathrm{m}$, double etch-pit tracks could be induced by proton recoils from neutron interactions or by low energy nuclei from muon interactions in the material surrounding the apparatus.

For the few candidates remaining after the analysis of the first sheet (an average of $5 / \mathrm{m}^{2}$ ), we looked for a coincidence in position, angles and RELs in the third (bottom) CR39 layer. The second foil was etched in $6 \mathrm{~N}$ $\mathrm{NaOH}$ water solution at $70{ }^{\circ} \mathrm{C}$ for 30 hours. An accurate scanning at high magnification was performed within an area of about $1 \mathrm{~mm}^{2}$ around the expected position of the candidate.

We estimate a global efficiency of the procedure of $99 \%$. No two-fold coincidence was found, that is no magnetic monopole candidate was detected. The $90 \%$ C.L. flux upper limits are at the level of $2.1 \times 10^{-16}$ $\mathrm{cm}^{-2} \mathrm{~s}^{-1} \mathrm{sr}^{-1}$ for $g=g_{D}$ magnetic monopoles with $\beta \sim$ $10^{-4}$ and $1.5 \times 10^{-16} \mathrm{~cm}^{-2} \mathrm{~s}^{-1} \mathrm{sr}^{-1}$ for magnetic monopoles with $\beta \sim 1$ (Fig. 8, curves "CR39").

\subsection{Combined search for fast magnetic monopoles}

A search for fast magnetic monopoles with scintillators or streamer tubes alone was affected by the background due to high energy muons with large energy losses in the detector. This background could be reduced by a combination of geometrical and energy cuts imposed on each of the sub-detectors. A combined analysis, based on all three subsystems, allowed the use of these cuts in a rather conservative way. Moreover, any systematic error was greatly reduced by the combination of measurements from the three subsystems. The redundancy and complementary offered by the MACRO detector allowed a good rejection power against the background and a high reliability of possible candidates. The analysis procedure is fully described in 14]. It used the data taken with the scintillator and the streamer tube sub-detectors to identify candidate events. This was done by reconstructing the energy release 


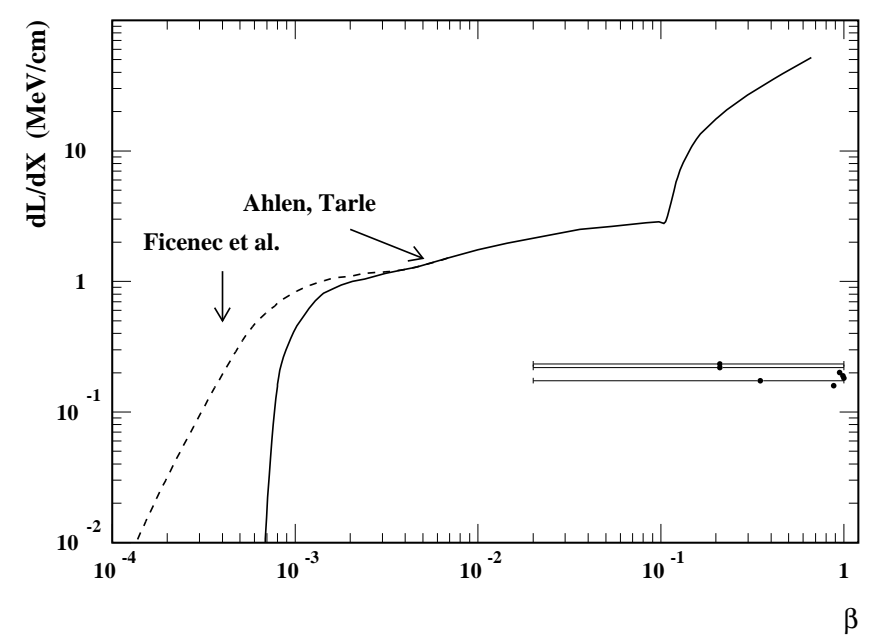

Fig. 7. Combined search: Light yield for the seven MM candidates which survived the scintillator and streamer tube cuts. The candidates light yields are compared with the expected magnetic monopole signal as computed in 15, 18 (see Sect. 2.4).

(using the scintillators' Energy Reconstruction Processor -ERP - and the streamer tube Charge and Time Processor - QTP [5.6]) and the particle's trajectory (using the streamer tubes' digital hit information). Any remaining events were then searched for in the track-etch layers as a final tool for their rejection or confirmation. The trigger selection criteria required at least one fired scintillation counter and 7 hits in the horizontal streamer planes of the lower subdetector [12]. Once the event tracking was performed, the value of the energy lost in the scintillator intercepted by the track was reconstructed using the ERP system. The reconstructed energy in each selected scintillation counter was required to be $\Delta E \geq(\Delta E)_{\min }=150 \mathrm{MeV}$. The minimum light yield by a MM in $10 \mathrm{~cm}$ pathlength in the scintillator is $\sim 230 \mathrm{MeV}$. A further selection was applied on the streamer tube pulse charge by using the multiple measurements provided by QTPs along the particle trajectory.

A cut 14 was applied on the event average streamer charge, by exploiting the logarithmic dependence of the streamer charge on the primary ionization [23,24].

In the analyzed data set, seven events survived the cuts. For these events the track-etch wagons identified by the streamer tracking system were extracted and analyzed. No track compatible with the crossing of a MM was found. As a further check, for each candidate, the measured value of the energy lost in the scintillation counters was compared with the expected signal of a magnetic monopole of the same velocity 15,18 . This comparison is shown in Fig. 7. For three of the seven events, the TOF information was provided by the streamer system alone, since only one scintillation counter was present. In this case the error on the reconstructed velocity is large because of the limited time resolution $(3 \mu \mathrm{s})$ of the streamer tubes. For all the events the measured energy losses were well below the expectations for a

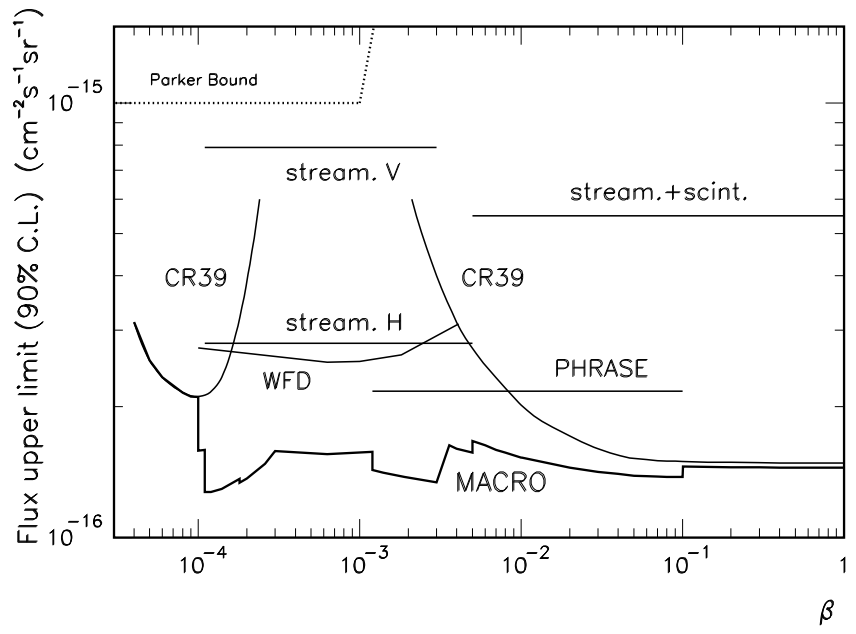

Fig. 8. Upper limits for an isotropic flux of magnetic monopoles obtained by the different MACRO analyses as described in the text. The global limit is presented as curve "MACRO".

MM. The analysis referred to about 4.8 live years; the maximum geometrical acceptance, computed by Monte Carlo methods, including all the analysis requirements, was $3565 \mathrm{~m}^{2}$ sr. The average global efficiency was $77 \%$.

Since no candidate survived, we set a $90 \%$ C.L. flux upper limit at $5.5 \times 10^{-16} \mathrm{~cm}^{-2} \mathrm{~s}^{-1} \mathrm{sr}^{-1}$ (presented in Fig. 8 as curve "stream. + scint.") for magnetic monopoles with $5 \times 10^{-3}<\beta<0.99$. The analysis ends at $\beta=0.99$ since for Lorentz factor $\gamma \gtrsim 10$ magnetic monopoles might induce showers in the detector, reducing the analysis efficiency 14.

\section{The global MACRO limit}

No magnetic monopole candidates were found in any of the above mentioned searches. Since any one of the sub-detectors may rule out, within its own acceptance and sensitivity, a potential candidate, a global limit was computed by combining together the limits obtained by the single analyses. The final MACRO limit and the limits obtained by the single subdetectors are given in Tab. 1 and shown in Fig. 8. Each search " $i$ " produced a $90 \%$ C.L. flux limit given by $\Phi_{i}=2.3 / A_{i}$, where $A_{i}$ is the analysis time integrated acceptance. In order to obtain the global MACRO limit, we divided the full $\beta$ interval in a number of slices sufficient to characterize the changes in the individual acceptances. We required that the significance of the global limit (in terms of C.L.) is not altered.

In order to illustrate the algorithm, suppose that in a specific $\beta$ slice there are two analyses, "1" and "2", based on the use of two sub-detectors during the same period of time. We consider analysis " 1 " as "dominant" in the sense that it contributes with its full time integrated acceptance $A_{1}$ to the global time integrated acceptance $A_{G}$. Analysis "2" then contributes to the global limit only with its independent parts relative to " 1 ". We consider 


\begin{tabular}{|c|c|c|c|c|c|c|c|}
\hline & \multicolumn{7}{|c|}{ Flux upper Limits $\left(10^{-16} \mathrm{~cm}^{2} \mathrm{~s}^{-1} \mathrm{sr}^{-1}\right)$} \\
\hline$\beta$ range & CR39 & WFD & Stream. H & Stream. V & PHRASE & Stream.+scint. & Global \\
\hline$(4.0 \div 10.0) \times 10^{-5}$ & $3.1 \div 2.1$ & & & & & & $3.1 \div 2.1$ \\
$(1.0 \div 1.1) \times 10^{-4}$ & 2.8 & 2.7 & & & & 1.6 \\
$(1.1 \div 2.6) \times 10^{-4}$ & $2.2 \div 7.5$ & 2.5 & 2.8 & 7.9 & & & $1.3 \div 1.5$ \\
$(2.6 \div 12.0) \times 10^{-4}$ & & 2.5 & 2.8 & 7.9 & & & 1.6 \\
$(1.2 \div 1.9) \times 10^{-3}$ & & $2.5 \div 2.6$ & 2.8 & 7.9 & 2.2 & & 1.4 \\
$(1.9 \div 3.0) \times 10^{-3}$ & $7.5 \div 3.9$ & $2.6 \div 2.9$ & 2.8 & 7.9 & 2.2 & & 1.3 \\
$(3.0 \div 4.1) \times 10^{-3}$ & $3.9 \div 3.1$ & $2.9 \div 3.1$ & 2.8 & 7.9 & 2.2 & & 1.6 \\
$(4.1 \div 5.0) \times 10^{-3}$ & $3.1 \div 2.8$ & & 2.8 & & 2.2 & 5.5 & $1.6 \div 1.66$ \\
$5.0 \times 10^{-3} \div 0.1$ & $2.8 \div 1.5$ & & & & 2.2 & 5.5 & $1.8 \div 1.5$ \\
$0.1 \div 1.0$ & 1.5 & & & & & 5.5 & 1.4 \\
\hline
\end{tabular}

Table 1. The $90 \%$ C.L. flux upper limits (in units of $10^{-16} \mathrm{~cm}^{-2} \mathrm{~s}^{-1} \mathrm{sr}^{-1}$ ) as a function of $\beta$ for an isotropic flux of $g=g_{D}$ magnetic monopoles with $m \geq 10^{17} \mathrm{GeV} / \mathrm{c}^{2}$. The limits discussed in Section 2 are given in columns two to six; the global MACRO limit discussed in Section 3 is given in the last column.

both the temporal independence as well as the spatial (geometric) independence versus the dominant analysis. The temporal independence is determined by comparing the "time efficiencies" $\epsilon_{i}^{t}$ of the analyses, defined as the ratios of each analyses live time to the covered solar time. If $\epsilon_{2}^{t}>\epsilon_{1}^{t}$, the coefficient representing the temporal independence of " 2 " versus " 1 " is $c_{2,1}^{t}=\epsilon_{2}^{t}-\epsilon_{1}^{t}$; otherwise, $c_{2,1}^{t}=0$. In the case of the track-etch subdetector, there is no dead-time, so its temporal efficiency is equal to 1. The coefficient representing the geometric independence of analysis " 2 " versus " 1 ", $c_{2,1}^{s}$, originates from the difference between the acceptances of the analyses. It is obtained by Monte Carlo simulations, assuming an incoming isotropic flux of magnetic monopoles with respect to subdetector "2": $c_{2,1}^{s}=\left(N_{2}-N_{1,2}\right) / N_{2}$, where $N_{2}$ and $N_{1,2}$ are the number of MMs detected by " 2 " and both analyses, respectively.

The global time integrated acceptance is then: $A_{G}=A_{1}+c_{2,1}^{t} A_{2}+\left(1-c_{2,1}^{t}\right) c_{2,1}^{s} A_{2}$ The global $90 \%$ C.L. limit for the flux of magnetic monopoles is $\Phi_{G}=2.3 / A_{G}$.

The algorithm used to combine the actual MACRO limits is more complicated than the example above. For each analysis we took into consideration its actual history, eliminating the longer periods of time in which it was eventually missing, and the changes in the detector configuration (super-modules involved). Those corrections were more critical in the case of earlier analyses, that were carried on during the construction of the MACRO detector and during initial tests; note that limits obtained by such older searches are not presented in Fig. 8 and in Tab.1, as they are considerably higher than the included ones, but they have still their imprint on the global limit.

In Fig. 9 we present the global MACRO limit; for comparison, the flux limits from other experiments which searched for magnetic monopoles with similar properties, are also shown [31]. In the figure the arrow indicates the Extended Parker Bound (EPB) at the level of $1.2 \times 10^{-16}$ $\left(\mathrm{m} / 10^{17}\right) \mathrm{cm}^{-2} \mathrm{~S}^{-1} \mathrm{sr}^{-1}$, which was obtained by consider-

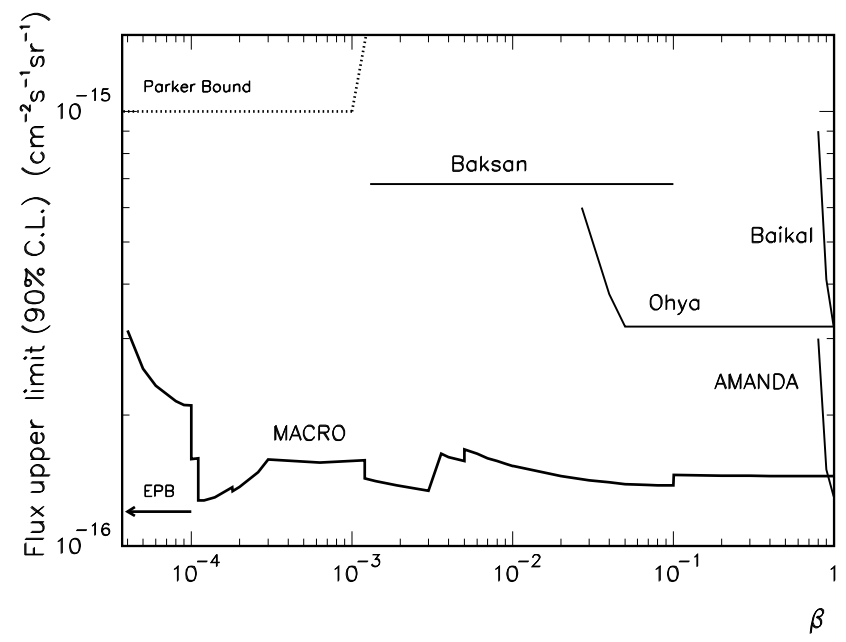

Fig. 9. The global MACRO limit for an isotropic flux of bare magnetic monopoles, with $m \geq 10^{17} \mathrm{GeV} / \mathrm{c}^{2}, g=g_{D}$ and $\sigma_{\text {cat }}<$ few mb. For comparison, we present also the flux limits from other experiments [31].

ing the survival probability of a magnetic monopole of mass $m$ in an early magnetic seed field 32.

\section{Discussion}

Our analysis applies to an isotropic flux of bare MMs with charge $g=g_{D}=\mathrm{e} / 2 \alpha$ and nucleon decay catalysis cross sections smaller than $1 \mathrm{mb}$ [1].

The magnetic monopole flux at the detector site is isotropic if magnetic monopoles have enough kinetic 


\begin{tabular}{|c|c|c|}
\hline$\Phi\left(\mathrm{cm}^{2} \mathrm{~s}^{-1} \mathrm{sr}^{-1}\right)$ & $\mathrm{m}\left(\mathrm{GeV} / \mathrm{c}^{2}\right)$ & $\beta$ \\
\hline$\leq 1.4 \times 10^{-16}$ & $\gtrsim 10^{10}$ & $>10^{-1}$ \\
$\leq 1.4 \times 10^{-16}$ & $\gtrsim 10^{16}$ & $>10^{-4}$ \\
$\leq 2.8 \times 10^{-16}$ & $\gtrsim 10^{6}$ & $>10^{-1}$ \\
$\leq 2.8 \times 10^{-16}$ & $\gtrsim 10^{10}$ & $>10^{-4}$ \\
\hline
\end{tabular}

Table 2. Final results of magnetic monopole searches with the MACRO experiment. The limits depend on the magnetic monopole mass and on its velocity.

energy (i.e. large mass and/or high $\beta$ ) in order to cross the Earth. If this is not the case, only a fraction of the total detector acceptance is actually exploited and the upper limits given above must be corrected accordingly. In particular, if monopoles have sufficient energy to cross the overburden mountain (i.e. $\sim 3700 \mathrm{hg} / \mathrm{cm}^{2}$ ) and reach the detector from above, but not enough to cross the Earth, the flux upper limit is about a factor of two weaker. Therefore the energy losses suffered by magnetic monopoles in traversing the Earth set the accessible values of mass and $\beta$ for a given experiment. For MACRO, at least one half of the geometrical acceptance is ensured for relativistic MMs with $m \gtrsim 10^{6} \mathrm{GeV} / \mathrm{c}^{2}$ or, for $\beta \simeq 10^{-4}$ if $m \gtrsim 10^{10} \mathrm{GeV} / \mathrm{c}^{2}$; the full acceptance is reached if $m \gtrsim$ $10^{10} \mathrm{GeV} / \mathrm{c}^{2}$ or $m \gtrsim 10^{16} \mathrm{GeV} / \mathrm{c}^{2}$, for relativistic and slow magnetic monopoles, respectively [33,34 (see Table 2). Sensitivity to fast and light MMs is also important, since they could be responsible for ultra high energy cosmic ray events above the Greisen-Zatsepin-Kuzmin cutoff [35].

If magnetic monopoles have $g>g_{D}$ and/or an associated electric charge (dyons [1]), the detection efficiency might change. As far as our sub-detectors are concerned, the dyon detection efficiency would be greater than that of bare monopoles since the excitation/ ionization-based energy losses would be larger [8]. The only exception is the detection of very slow dyons with the streamer tubes, since it relies on the Drell effect on helium atoms [22], which for dyons might be prevented by coulombian repulsion. However, as shown in [8], the only effect would be the raising of the minimum velocity threshold at $\beta \simeq\left(1.7 \times 10^{-4}\right) \sqrt{Z}$ for dyons with electric charge $Z e$, the threshold for bare MMs being $\beta=1.1 \times 10^{-4}$. As suggested in [36], GUT magnetic monopoles may catalyze nucleon decays along their path with a cross section $\sigma_{c}$ of the order of the hadronic cross sections [1]. If $\sigma_{c} \gtrsim 1 \mathrm{mb}$, the efficiencies of the aforementioned searches might decrease due to the effects of the fast decay products. A deep study was performed on the effect of the nucleon catalysis cross section on the streamer tube analysis. The main result is that the present analyses are still efficient up to at least $\sigma_{c} \simeq 100 \mathrm{mb}$. A dedicated search for magnetic monopoles accompanied by one or more nucleon decays along their path was also performed. The results are reported in a separate paper [37].

\section{Conclusions}

We present the final results of GUT magnetic monopole searches performed with the MACRO detector at Laboratori Nazionali del Gran Sasso (Italy). Different searches using the MACRO sub-detectors (i.e. scintillation counters, limited streamer tubes and nuclear track detectors) both in stand alone and combined ways, were performed. Since no candidates were detected, Tab.2 summarizes the 90\% C.L upper limit to an isotropic flux of bare MMs with charge $g=g_{D}=\mathrm{e} / 2 \alpha$ and nucleon decay catalysis cross section smaller than $1 \mathrm{mb}$.

\section{Acknowledgements}

We gratefully acknowledge the support of the director and of the staff of the Laboratori Nazionali del Gran Sasso and the invaluable assistance of the technical staff of the Institutions participating in the experiment. We thank the Istituto Nazionale di Fisica Nucleare (INFN), the U.S. Department of Energy and the U.S. National Science Foundation for their generous support of the MACRO experiment. We thank INFN, ICTP (Trieste), WorldLab and NATO for providing fellowships and grants for non Italian citizens.

\section{References}

1. J. Preskill, Ann. Rev. Sci. 34 (1984) 461;

G. Giacomelli, Riv. Nuovo Cimento 7 (1984) 1;

D. E. Groom, Phys. Rep. 140 (1986) 324 and references therein.

2. S. F. King and Q. Shafi, Phys. Lett. B422 (1998) 135;

T. W. Kephart and Q. Shafi, hep-ex/0105237 (2001).

3. T. W. Kephart and T. J. Weiler, Astrop. Phys. 4 (1996) 217

C. O. Escobar and R. A. Vazquez, Astrop. Phys. 10 (1999) 197.

4. M. S. Turner et al., Phys. Rev. D26 (1982) 1296.

5. S. P. Ahlen et al. (MACRO Coll.), Nucl. Instr. \& Meth. in Phys. Res. A324 (1993) 337.

6. M. Ambrosio et al. (MACRO Coll.), NIM A486 (2002) 663.

7. M. Ambrosio et al. (MACRO Coll.), Astropart. Phys. 10 (1999) 11, hep-ex/9807009.

8. J. Derkaoui et al., Astropart. Phys. 10 (1999) 339.

9. M. Ambrosio et al. (MACRO Coll.), Phys. Lett. B406 (1997) 249.

10. S. P. Ahlen et al (MACRO Coll.), Phys. Rev. Lett. 72 (1994) 608.

11. M. Ambrosio et al.(MACRO coll.), Astropart. Phys. 6 (1997) 113.

12. M. Ambrosio et al. (MACRO coll.), Astropart. Phys. 4 (1995) 33.

13. L. Patrizii (MACRO Coll.), Proc. of the XXth ICNTS, Rad. Meas. 34 (2001) 259.

14. M. Ambrosio et al. (MACRO Coll.), hep-ex/0110083, accepted by Astroparticle Physics.

15. D. J. Ficenec et al., Phys. Rev. D36 (1987) 311.

16. S. P. Ahlen, Phys. Rev. D17 (1978) 229. 
17. S. P. Ahlen and K. Kinoshita, Phys. Rev. D26 (1982) 2347.

18. S. P. Ahlen and G. Tarlé, Phys. Rev. D27 (1983) 688.

19. S. Kyriazopoulou (MACRO Coll.), Proc. NATO ARW on Cosmic Radiations, Oujda (Morocco) 21-23 March 2001;

S. Kyriazopoulou, Ph.D. Thesis, California Institute of Tecnology (2002).

20. S. P. Ahlen et al. (MACRO Coll.), Astropart. Phys. 1 (1992) 11.

21. A. Baldini et al., Nucl. Instr. \& Meth. in Phys. Res. A305 (1991) 475.

22. S. D. Drell et al., Phys. Rev. Lett. 50 (1983) 644. V. Patera, Phys. Lett. A137 (1989) 259.

23. G. Battistoni et al., Nucl. Instr. \& Meth. in Phys. Res. A270 (1988) 185.

24. G. Battistoni et al., Nucl. Instr \& Meth. in Phys. Res. A401 (1997) 309.

25. I. De Mitri et al., Nucl. Instr. \& Meth. in Phys. Res. A360 (1995) 311.

26. G. Battistoni et al., Nucl. Instr \& Meth. in Phys. Res. A399 (1997) 244.

27. S. Cecchini et al., Riv. Nuovo Cimento A109 (1996) 1119.

28. S. Cecchini et al. Radiat. Meas., 34 (2001) 54.

29. G. Giacomelli et al., Nucl. Instr \& Meth. in Phys. Res. A411 (1998) 41.

30. B. Choudhary (MACRO Coll.), $26^{\text {th }}$ ICRC, Salt Lake City, Utah, USA (1999), hep-ex/9905023;

M. Sitta (MACRO Coll.), $27^{t h}$ ICRC, Hamburg, Germany (2001).

31. E. N. Alexeyev et al. ("Baksan"), $21^{\text {st }}$ ICRC, Adelaide, Vol. 10, (1990) 83;

S. Orito et al. ("Ohya"), Phys. Rev. Lett. 66 (1991) 1951;

V. A. Balkanov for the Baikal Coll. ("Baikal", "AMANDA"), Nucl. Phys. B91 (2001) 438;

G. Giacomelli et al., hep-ex/0005041 (2000).

32. F.C. Adams et al., Phys. Rev. Lett. 70 (1993) 2511.

33. I. De Mitri, Ph.D. Thesis, University of L'Aquila (1996).

34. J. Derkaoui et al., Astropart. Phys. 9 (1998) 173.

35. T. W. Kephart and T. J. Weiler, Astropart. Phys. 4 (1996) 271

P. Bhattacharjee and G. Sigl, Phys. Rep. 327 (2000) 109;

S. D. Wick et al., astro-ph/0001233 and references therein. 36. V. A. Rubakov, JETP Lett. 33(1981) 644;

C. G. Callan, Phys. Rev. D25 (1982) 2141.

37. M. Ambrosio et al. (MACRO Coll.), Search for $\mathrm{Nu}$ cleon Decay induced by GUT Magnetic Monopoles with the MACRO Experiment, hep-ex 0207024. 